\title{
XII.
}

\section{Hysterische Pupillen- und Accommodationslähmung, geheilt durch hypnotische Suggestion.}

\author{
Von \\ Dr. Julius Donath \\ in Budapest.
}

Mit 8 Abbildungen.

Lähmungen im Gebiete des N. facialis, sowie der Binnenmusenlatur des Auges gelten allgemein als Beweise für anatomische Läsionen des Nervensystems, und das Vorhandensein der einen oder der anderen dieser Bewegungsstörungen hat bisher genügt, um die Annahme einer hysterischen, also "functionellen" Erkrankung fallen zu lassen.

Beztiglich der Facialislähmung hat $\mathrm{Charcot}$ durch lange Jahre die fast allgemein angenommene Lehre verkündet, dass b e i d e $r$ hysterischen Hemiplegie nie der untere Facialis derselben Seite betheiligt ist, wie es bei dengewöhnlichen Hemiplegien der Fall ist. Diesen seinen Satz brachte er selbst in jingster Zeit durch eine Beobachtung za Falle. ${ }^{1}$ ) Es handelte sich um einen 24 jährigen Mann, von beiden Eltern neuropathisch belastet, Potator, der ein mit heftigem Schreck einhergehendes Trauma auf dem Unterkiefer erlitten hatte. Ungefähr 2 Monate danach bekam er den ersten hystero-epileptischen Anfall, und es trat Lähmung des M. buccinatorius und zygomaticus major mit Anästhesie dieser Gegend auf. Demzufolge steht $\mathrm{Charcot}$ nicht mehr an, die Facialislähmung in den Fällen von Ballet, Chantemesse und Bonnet gleichfalls als hysterisch anzuerkennen. - Immerhin sind solche Facialislähmungen höchst selten. ${ }^{2}$ )

1) Archives de neurologie. Juli 1891 .

2) Vgl. auch J. Grasset, Traité pratique des maladies du système nerveux. Paris 1881. Hystérie, p. 943: „La paralysie peut atteindre un côté de la face, mais c'est très rare". Ferner: A. Str ümpell, Lehrbuch der speciellen Pathologie and Therapie der inneren Krankheiten. II. Bd. I. Thl. Leipzig 1884. Die Hysterie. S. 424: "Lähmungen in den Gesichtsmuskeln kommen fast niemals vor." 
Gilt aber das Freisein des Facialis für eine grosse Regel bei der Hysterie, so wird das Verschontbleiben des Sphincter iridis und M. ciliaris als ausnahmsloses Gesetz betrachtet. Mir ist wenigstens bisher aus der Literatur kein Fall von hysterischer Pupillen- und Accommodationslähmung bekannt geworden.

Die hysterische Natur dieser Läbmung ist aber, wie sich im Folgenden zeigen wird, durch den besonderen Charakter derselben und durch sonstige Begleiterscheinungen hysterischer Art, nicht minder auch durch die Heilungsweise genügend sichergestellt.

Bei diesem Individuum wurden anch sonst, wie ich gleich hier andeuten will, ausserordentlich merkwtirdige Erscheinungen in der Hypnose beobachtet, bezw. durch Snggestion hervorgerufen, und die Resultate dieser Studien durch strenge Controle ausser Zweifel gestellt. Da letztere nicht direct zur Krankengeschichte gehören, sondern mehr zur Bestärkung der Auffassung dieses Krankheitsbildes dienen sollen, so mögen dieselben zum Schluss kurz angefuhrt werden. Eine ausfubrlichere Darlegung derselben, welche auf manche strittige Punkte der Hysterie und des Hypnotismus einiges Licht verbreiten diurften, will ich zum Gegenstand einer besonderen Mittheilung machen.

J. K., 26 Jahre alt, ledig, Sprachlehrerin. Laut selbstgeschriebener Anamnese leidet der Vater an chronischem Gelenkrheumatismus und Herzaffection; Mutter an Herzschlag gestorben. Grossvater väterlicherseits an Nierenentzundung gestorben; Grossmutter gesund. Ueber die Todesursache der Grosseltern muitterlicherseits weiss sie nichts Näheres anzugeben. Von 4 Geschwistern 1 Bruder an Lungenemphysem leidend, die übrigen gesund.

Pat. batte im Jahre 1871 Masern, 1872 Keuchhusten, 1874 Windpocken, 1875 Scharlach, 1881 Lungenentzündung, 1882 gastrisches Fieber, 1883 ,Typhus (Gehirnentzündung infolge angestrengter Nachtarbeit bei den Vorbereitungen für die Prüfungen). Als Folge des Gehirnfiebers starke Kurzsichtigkeit und grosse Nervosität". 1885 schwerer Gelenkrheumatismus, ,der noch jetzt von Zeit zu Zeit auftritt, aber in schwachem Maasse". 1888 traten unter starken Fiebererscheinungen Schmerzen, Schwellungen und Entzündungen in der Wirbelsänle auf, welche von den behandelnden Aerzten für Beinhautentzündung erklärt wurden (innerlich Jodkalium, äusserlich Jodtinctur, Chapman'scher Schlauch und Quecksilbersalbe, welch' letztere Wunden erzeugte). „Man setzte diese Behandlung fort, bis ich so schwach wurde, dass ich fast gar nicht mehr gehen konnte. Dann endlich behandelten mich die Herren Dr. Do n a th mit Electricität und Dr. N. Reich mit Massage und Hängen, so dass ich ganz gesund wurde."

(Ich schalte hier ein, dass sich Pat. mir das erste Mal am 25. Februar 1889 in ziemlich herabgekommenem Zustande präsentirte. Ueber den Dornfortsätzen der Brustwirbel und der unteren, besonders 6. und 7. Nackenwirbel Röthung, Schwellung und starke Druckschmerzhaftigkeit. Gleichzeitig klagte sie über Rothsehen. Mit Weglassung jeder Medication wurde, 
Hyster. Pupillen- u. Accommodationsiähm., geheilt durch hypnot. Suggestion. 219

unter Anwendung der genannten physikalischen Heilmethoden, anf die Hebung der Ernäbrung das Hauptaugenmerk gerichtet und Pat. nach einigen Monaten wesentlich gebessert $u$. in trefflichem Ernährungszustande entlassen.

Seither hatte sie aber noch häufig heftige Rückenschmerzen.

Infolge von Influenza hatte sie durch 14 Wochen Lungenkatarrh mit starkem Husten. Ostern 1891 hustete sie wiederum, dabei zweimal eine beträchtliche Menge reinen Blutes. Da sie damals auch an Nachtschweissen litt, bekam sie vom behandelnden Arzt Atropin, und seither leidet sie an „,zeitweiliger Erweiterung und Lähmung der Pupillen“. Anfangs waren beide Pupillen gelähmt, später abwechselnd die eine oder die andere.

„Seit Ostern hatte ich bis zum Juli fast jede Woche Ohnmachtsanfälle und Nervenzuckungen, die fast regelmässig Sonntags wiederkehrten. Dann litt ich an Krämpfen im Halse und im Schlunde, die mich oft im Sprechen hemmten, da ich von Schluchzen unterbrochen wurde und zeitweise meine eigene Stimme wie aus weitester Ferne hörte, während ich das Sprechen Anderer deutlich vernahm. Ich litt an absolutester Appetitlosigkeit, hatte häufig Stechen im Körper, namentlich am Herzen."

„Alle diese Symptome haben fast gänzlich nachgelassen, seitdem Herr Dr. Donath mich seit dem Juli in hypnotischer Behandlung hat."

$\mathrm{Zu}$ dieser Anamnese der Pat. füge ich hinzu, dass zur Zeit, als sie zum ersten Male in meiner Behandlung stand, viel über Kopfschmerz, der 24-48 Stunden anzudauern pflegte, und leichte Gemüthserregbarkeit geklagt wurde. Trotzdem sie, wie erwähnt, ihre eigene Stimme wie von weiter Ferne und ihren Athem wie Röcheln zu hören vorgab, war objectiv eine gute Hörschärfe nachzuweisen. Dabei hänfiger Schwindel, Flimmern vor den Augen, ,wie ein Kaleidoskop".

$\mathrm{Zu}$ wiederholten Malen konnte ich bei ihr das spontane Auftreten von Blutextravasatchen unter den Fingernägeln in der Gestalt feiner, isolirter, etwa $1 / 2 \mathrm{Mm}$. langer, braunrother Linien beobachten, welche stets der Längaxeder $\mathrm{Nägel}$ parallel standen. Gewöhnlich traten sie unter allgemeinen Erregungszuständen auf, die oft einen Tag früher sich kundgaben, wobei über heftige Schmerzen in den Fingerspitzen geklagt wurde. Pat. machte mich dann oft auf das bevorstehende Auftreten dieser Strichelchen aufmerksam, was auch stets am nächsten Tage erfolgte. Nach ungefähr eintägigem Bestande schwanden sie und mit ihnen auch die Schmerzen. Diese Ekchymosen unter den Fingernägeln kommen noch heute vor, doch ohne besondere Schmerzen. ${ }^{1}$ ) Auch finden fortwährend Abschilferungen der Fingernägel statt (quergestellte, weisse, rauhe, 1-2 Mm. dicke Streifen, welche fast die ganze Breite der Fingernägel einnehmen).

Es sind ferner verzeichnet zeitweise, besonders nach Aufregungszuständen und schlaflosen Nächten auftretende Zuckungen im Gesichte, und in der letzteren Zeit Weinkrämpfe und eine Reihe hystero-epileptischer Anfälle.

Status praesens am 29. Juli 1891: Mittelgross, schwach von Knochenban und Musculatur, ziemlich schlecht genährt. Supraclavicular-

1) Im Laufe des vergangenen December und Januar wurde endlich auch zu wiederholten Malen das spontane Hervortreten von Blutstropfen unter den Fingernägeln beobachtet. 
gruben etwas eingesunken; Thorax schmal; Mammae schwach entwickelt. Lungenschall oben kurz, doch kejne Dämpfung; etwas rauhes Inspirium. Herztöne rein. - Linke Pupille zeigt normales Verhalten. Rechte Pu pille erweitert, a uf Licht und Accommodation nichtreagirend. Am linken Auge gute Farbenperception; am rechten werden die Nuancen etwas weniger deatlich unterschieden. A uf der ganzen rechten Körperhälfte - also auf der Seite der Pupillenlähmung - Tast-und Schmerzempfindung stark herabgesetzt, besonders auf der rechten Brust- und Bauchhälfte, wo Berübrung und leichte Nadelstiche gar nicht empfunden werden. Klagt über sehr gedrückte Gemüthsstimmung, vollständige Appetitlosigkeit und Widerwillen gegen Speisen, über andauernde Schlaflosigkeit, trotz mannigfacher Schlafmittel, und Kribbeln in den Extremitäten.

Es wurde die Diagnose auf hysterische Pupillen- und Accomodationslähmung gestellt und mit der hypnotischen Behandlung sofort begonnen.

Pat., welche früher nie hypnotisirt wurde, geräth durch einfache Suggestion des Schlafes und Auflegen meiner Hand auf Augen und Stirn nach wenigen Minuten in Hypnose, welehe gleich das erste Mal ebenso vollständig war, wie in allen folgenden Sitzungen.

Zu Beginn der Hypnose zeigten sich bei ihr, besonders in den ersten Sitzungen, lebhafte clonische Schlingkrämpfe, sowie Krämpfe der Halsmuskeln, zuweilen auch Gesichtskrämpfe (Clonus des Corrugator supercilii beiderseits, des linken Levator labii super. alaeque nasi und auch der ganzen linken Wangenmusculatur). Doch liessen dieselben im Laufe der Hypnose bald wieder nach, und infolge darauf gerichteter suggestiver Einwirkung treten dieselben gegenwärtig überhaupt nur mehr selten auf, mit Ausnahme leichter Schlingkrämpfe, welche noch jetzt die Hypnose einleiten. Desgleichen ist das Gefühl von Stechen in der Herzgegend, worüber sie in der ersten Zeit zu Beginn jeder Sitzung geklagt hatte, jetzt gänzlich weggeblieben. - Der Eintritt der Hypnose ist durch vollkommene Flexibilitas cerea scharf markirt. In jeder Hypnose wurde das Aufhören der ihr nambaft gemachten Krankheitserscheinungen (Pupillenlähmung, Krämpfe, Anästhesie, Appetit- und Schlaflosigkeit, traurige Verstimmung u.s. w.) und die baldige Heilung suggerirt. Beim Er wachen, wie ich mich zu wiederholten Malen genau überzeugt habe, vollständige Amnesie, doch weiss sie in der Hypnose bis ins kleinste Detail Alles genau anzugeben, was in allen früheren, oft längst vergangenen Hypnosen geschehen ist. - Das Erwachen erfolgt gleichfalls auf einfache Suggestion nach einer kleinen Weile, wobei im Mienenspiel, im schweren Oeffnen der Lider und in den langsamen Bewegungen des Körpers sich eine gewisse Anstrengung ausdrückt, den hypnotischen Zustand zu überwinden.

Während des ganzen hypnotischen Heilverfahrens kam absolut kein anderes Mittel zur Verwendung.

31. Juli. Nach 2 hypnotischen Sitzungen Schlaf, Appetit besser, Stimmung heiter. Rechte Brust- und Bauchhälfte, sowie Unterextremität fast ebenso sensibel wie links. Rechte Oberextremität zeigt noch herabgesetzte Empfindung. Herr College W. Goldzieher constatirt eine geringe Verengerung der rechten Pupille und Abnahme der Accommodationslähmung. Hypnotische Suggestion. 
Hyster. Pupillen- a. Accommodationslähm., geheilt durch hypnot. Suggestion. 221

8. Angust. Rechte Pupille normal. Dagegen ist die linke Pupille erweitert und reagirt nicht auf Licht. Desgleichen ist jetzt die Sensibilitat auf der rechten Oberextremität normal geworden, während sie auf der linken herabgesetzt ist. ${ }^{1}$ )

9. August. Linke Pupille stark erweitert, auf Licht nicht reagirend; Accomodationslähmang. Schmerzempfindung linkerseits auf beharter Kopfhant; Gesicht, Halsund 0 berextremität schwächer als rechts. Dagegen ist dieselbe auf Brust, Bauch, Unterextremitäten beiderseits gleich.

M. Mohr:

11. August. Augenuntersuchung durch Herrn Assistenzarzt Dr.

Rechtes Auge. M. 16,0 D, V $=6 / 18 ;$ Nahepunkt $50 \mathrm{Mm}$., liest Schweigg. III Linkes $=$ M. $10,0 \mathrm{D}, \mathrm{V}=6 / 36 ; \quad \Rightarrow 140=\leq$ IV Rechte Pupille mittelweit, ziemlich gut reagirend; linke Pupille über mittelweit weder auf Licht, noch auf Accomodation reagirend. Die Untersuchung mittelst des M ey erhausen'schen selbstregistrirenden Perimeters zeigt an beiden Augen, besonders am linken, concentrische Gesichtsfeldeinschränkung. Das Gesichtsfeld betrug für Weiss am rechten Auge: oben $40^{\circ}$, oben aussen $36^{\circ}$, anssen $45^{\circ}$, aussen unten $45^{\circ}$, unten $45^{\circ}$, unten innen $32^{\circ}$, innen $40^{\circ}$, innen oben $40^{\circ}$. - Am linken Auge in derselben Reihenfolge: $37^{\circ}, 27^{\circ}, 24^{\circ}, 18^{\circ}, 27^{\circ}, 20^{\circ}, 25^{\circ}, 22^{\circ}$.

Schon am folgenden Tage (12. August) wird am rechten Auge die Pupille über mittelweit und nicht reagirend gefunden; Nahepunkt in $150 \mathrm{Mm}$., gelesen wird Sehweigger IX. Gesichtsfeld beider Augen zeigt für Weiss ungefähr gleichmässige, ebenso starke concentrische Eiuschräukung, wie gestern das des linken Auges. Das Gesichtsfeld betrug nämlich für Weiss am rechten Auge: $25^{\circ}, 20^{\circ}, 23^{\circ}, 19^{0}, 25^{\circ}, 23^{\circ}, 26^{\circ}, 24^{0}-\mathrm{am}$ linken Auge: $25^{\circ}, 24^{\circ}, 19^{\circ}, 20^{\circ}, 20^{\circ}, 20^{0}, 20^{\circ}, 170$. - Ebenso zeigte sich beiderseits starke concentrische Gesichtsfeldeinengung für Farben, und zwar hatte Roth am rechten Ange eine minimale und maximale Ausdeh-

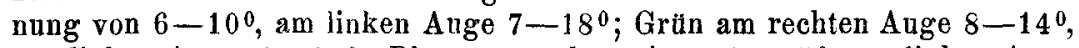
am linken Ange $4-13^{\circ} ;$ Blan am rechten Auge 8-150, am linken Auge $7-16^{0}$. (Siehe Fig. $1-4$ S. 222 u. 223.)

Die Angabe der Pat., dass sie solche rasch wechselnde Lähmungs. zustände der Pupillen schon früher an sich wahrgenommen, wird also durch diese und die folgenden Beobachtungen gestützt. - Am selben Tage (12. August) an beiden Oberextremitäten in gleicher Weise herabgesetzte

1) Ich will hier ein für allemal bemerken, dass bei der ernsten, intelligenten Kranken, welche zu mir gekommen war, um möglichst bald geheilt und erwerbsfähig gemacht $\mathrm{zu}$ werden, meinerseits jeder Schein vermieden wurde, dass ich für das eine oder das andere Symptom besonderes Interesse habe; kein Befund wurde ihr mitgetheilt, und sie erfuhr nichts von alledem, was in der Hypnose vorgegangen ist. Es sind dies ebenso selbstverständliche, wie bäufg genug vernachlässigte Cautelen gegen unbewusste Suggestionen Seitens des Arztes und Autosuggestionen Seitens der Kranken, die leicht eine bohe Meinung von der Wichtigkeit ibres $\mathrm{Zu}$ standes bekommen können. 
12. August 1891. Beiderseitige Pupillen- und Accommodationslähmang.

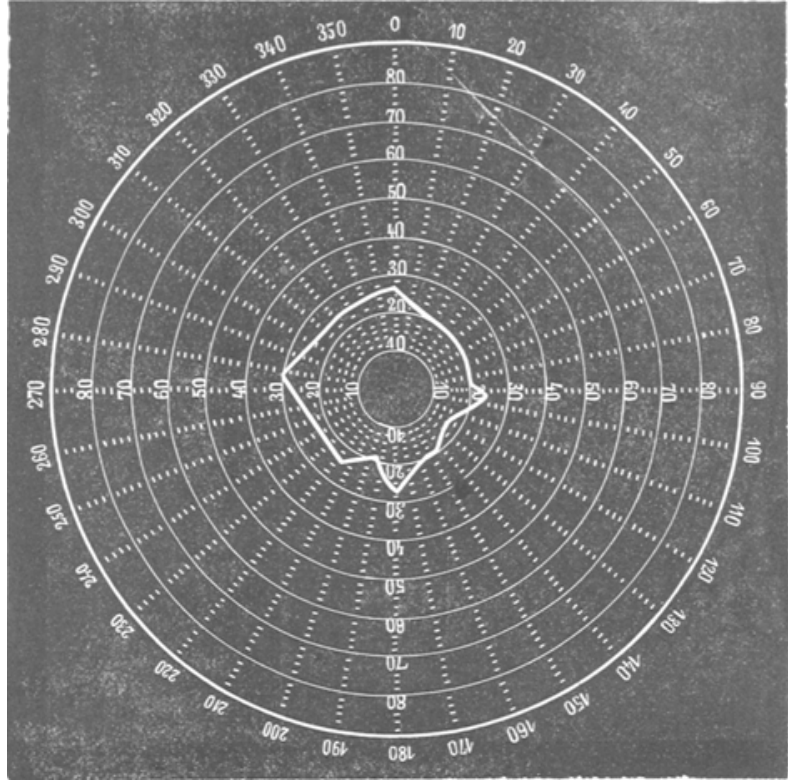

Fig. 1. Rechtes Auge: Weiss.

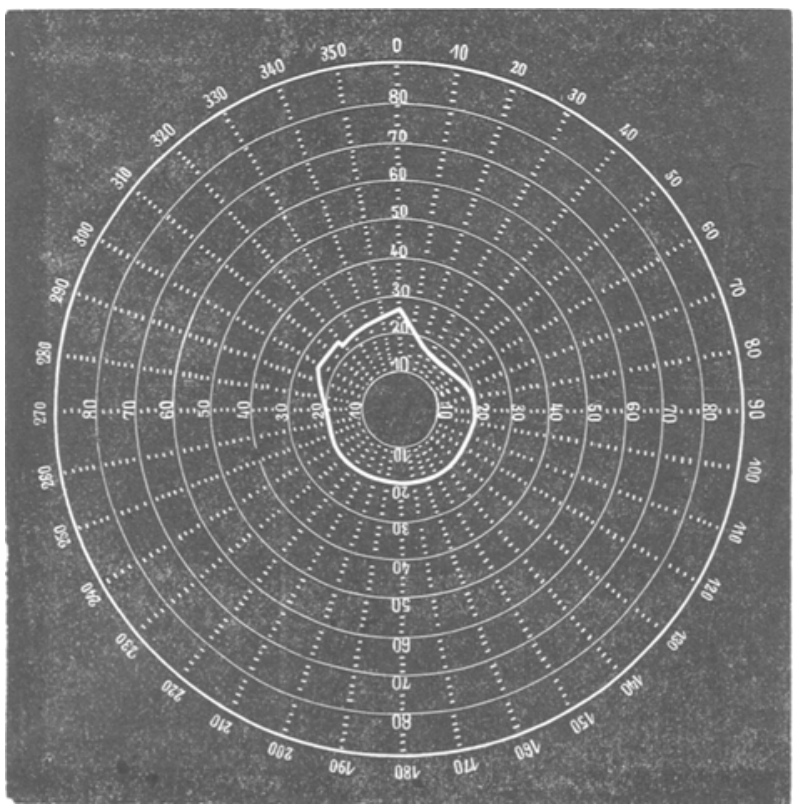

Fig. 2. Linkes Auge: Weiss. 
Hyster. Pupillen- u. Accommodationslähm., geheilt durch hypnot. Suggestion. 223

12. August 1891. Beiderseitige Pupillen- nnd Accommodationslähmung.

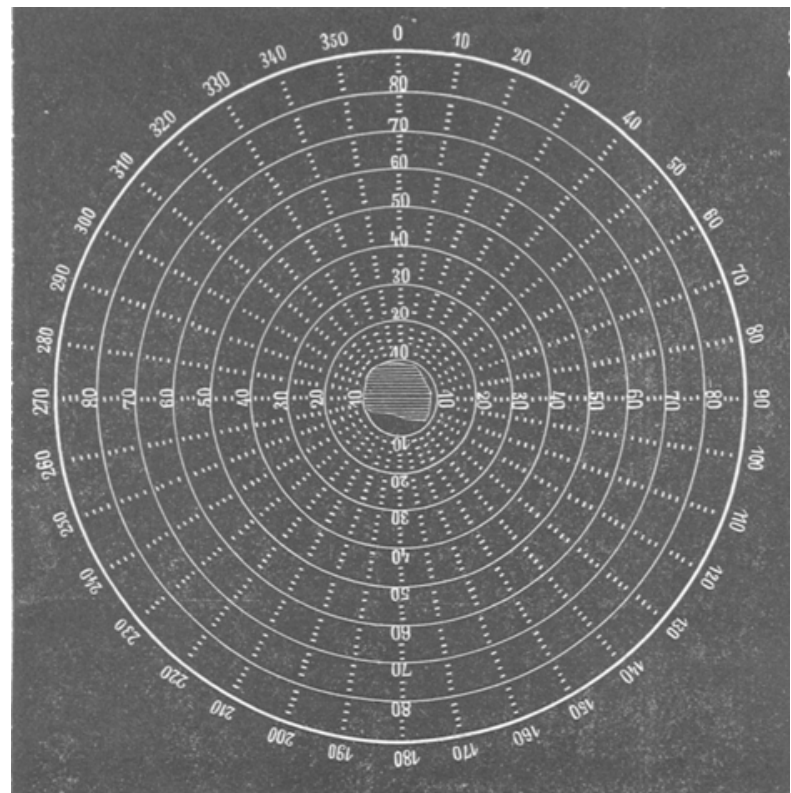

Fig. 3. Rechtes Auge: Roth.

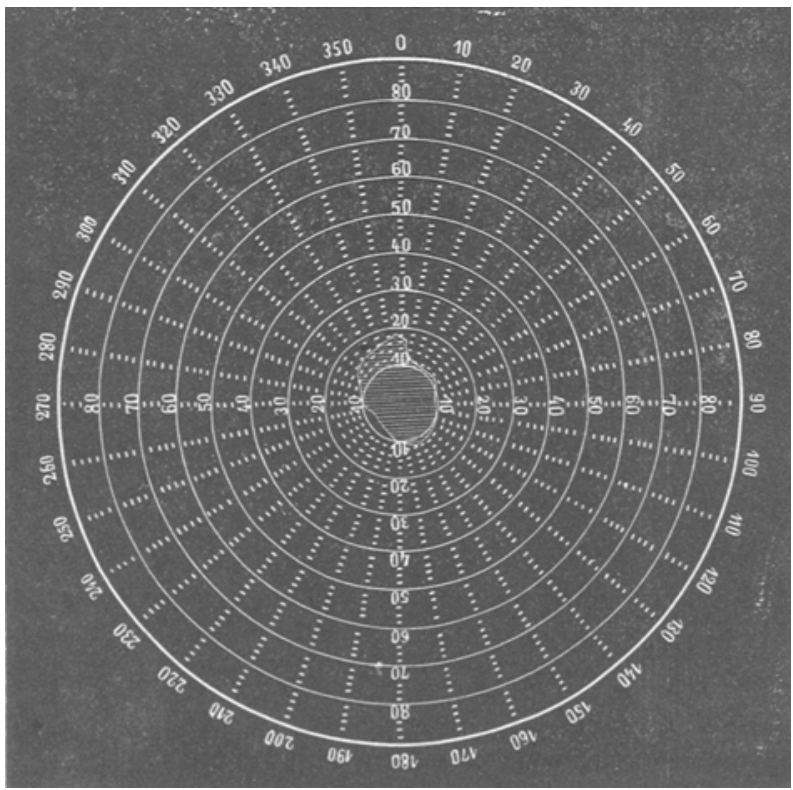

Fig. 4. Linkes Auge: Roth. 
Tast- und Schmerzempfindung. Auch ist die ganze behaarte Kopfhaut gegen Nadelstiche unempfindlich. Dagegen zeigen Gesicht, der ganze Rumpf, die Beine gute Tast- und Schmerzempfindung.

19. A ugust. Beide Pupillen bedeutend enger. Die hysteroepileptischen Anfälle, Weinkrämpfe haben seit Beginn der hypnotischen Behandlung gänzlich aufgehört. Doch hat sie noch Abends, wenn sie sich niederlegt, leichte Schlingkrämpfe, gleichwie es zu Beginn der Hypnose der Fall ist. Zeigt ein ruhigeres Benehmen, Gemüthsstimmung, Appetit, Schlaf anhaltend gebessert, wie auch von der Umgebung bestatigt wird.

20. August. Beiderseits vollständige Pupillen-und Accommodationslähmung.

21. August. Papillen etwas enger. Anästhesie am ganzen Körper - auch die der behaarten Kopfhant - gänzlich geschwunden.

27. August. Pupillen enger, gleich weit; linke reagirt auf focale Beleuchtung, rechte jedoch nicht.

31. A ugust. Um meine therapeutische Suggestion durch Autosuggestion Seitens der Kranken zu verstärken, fragte ich sie in der Hypnose, wie lange die Krankheit dauern werde. Sie antwortete: „Noch ziemlich lange, einen Monat". - Dementsprechend wurde ihr auch in der Folge zu wiederholten Malen sowohl im hypnotischen, als auch im wachen $\mathrm{Zu}$ stande suggerirt, dass die Cur zu Ende des Monates vollendet sein werde.

3. September. Pat. berichtet hieute, dass die Pupillen des Morgens ganz enge waren und im Laufe des Tages allmählich wieder weiter wurden. Ich fand beide (4 Uhr Nachmittags) mässig erweitert, auf Licht nicht reagirend. - Ich bemerke hier, dass eine in der heutigen Hypnose eindringlich vorgenommene Suggestion, der zufolge die Pupillen beim Erwachen normal sein werden, sich als wirkungslos erwiesen hat.

10. September. Befund des Herrn Collegen W. Goldzieher: „Beiderseits Pupillen- und Accommodationslähmung. Fernpunkt des linken Auges in ca. $16 \mathrm{Cm}$, des rechten Auges in ca. $13 \mathrm{Cm}$. Schweigg. I kann nur in dieser Entfernung gelesen werden; um näher gerückt zu werden, müssen bereits Convexgläser benutzt werden. Beiderseits bedeutende concentrische Gesichtsfeldeinengung, für Weiss ca. 200, für Roth and Grün ca. $10-15^{0} \mathrm{im}$ horizontalen und verticalen Diameter."

12. September. Tast-, Schmerz- und Temperaturempfindung am ganzen Körper normal, nur Stirn- und behaarte Kopfhaut sind, trotz energischer Suggestion, bis gegenwärtig (22. November) anästbetisch geblieben. (Tiefe Nadelstiche werden als Berührung, Berührung überhaupt gar nicht empfunden.)

14. September. Leichte Schlingkrämpfe treten nur noch zu Beginn und während der Hypnose auf, sonst ist sie frei davon.

19. September. Klagt heute wieder über Schmerzen in den mittleren Brustwirbeln, welche druckempfindlich sind. In der Hypnose wird ihr das Verschwinden der Rückenschmerzen mit den übrigen Symptomen suggerirt.

2. October. Pat. meldet mir Nachmittags freudig, dass die Papillen verengt sind und sie gut lesen kann. Nach Dr. Goldzieher: "Pupillen auf Lichteinfall vollkommen beweglich; Accom- 
modation hergestellt. Auch die Besserungin Bezug auf die Einengung des Gesichtsfeldes muss als eine a uffallendebezeichnet werden." Der Befund wurde am 10. October auch vom Herrn Collegen A.Szili bestätigt. - Sie schläft jede Nacht 5 Stunden, und ist das Befinden auch im Uebrigen ein vollständig befriedigendes. Vom Beginn der Behandlung bis Ende September haben 24 hypnotische Sitzungen stattgefunden, und ist die Heilung der für Ende September anberaumten Pupillenlähmung am 2. October erfolgt.

19. October. Seit gestern wieder beiderseits Pupillen- und Accommodationslähmung, was Pat. mir besturzt meldet. Hypnotische Suggestion, dass die Lähmung der Pupillen, sowie die Unempfindlichkeit der Stirnand behaarten Kopfhaut in den nächsten Tagen schwinden werden.

21. October. Rechte Pupille enger, auf Licht reagirend. Linke Pupille unverändert gelähmt.

25. October. In der Hypnose befragt, wann die Pupillenlähmung aufhören werde, antwortet sie: ,Ende October", weil diese von den Rückenschmerzen abhänge, die dann auch aufhören würden.

30. October. A u ch die linke Pupille, welche schon vorgestern etwas enger war und auf Licht schwach reagirte, ist he ute $n$ ur wenig weiter als die rechte und zeigt prompte Lichttreaction.

22. November. Pupillenreaction und Accommodation beiderseits seither anhaltend normal. Die perimetrische Untersuchung durch Herrn Collegen A. Szili ergab für Weiss am rechten Ange: oben $40^{\circ}$, oben aussen $32^{\circ}$, aussen $33^{\circ}$, aussen unten $30^{\circ}$, unten $45^{\circ}$, unten innen $33^{\circ}$, innen $34^{\circ}$, innen oben $33^{\circ}$. Am linken Auge in derselben Reihenfolge $50^{\circ}, 540,55^{0}, 60^{\circ}, 55^{\circ}, 58^{\circ}, 50^{\circ}, 39^{\circ}$. - Desgleichen sind die Gesichtsfelder für Farben erweitert: Grün zeigt eine minimale und maximale Ausdehnung am rechten Auge von $14-23^{\circ}$, am linken Auge $7-27^{\circ}$; Roth am rechten Auge 25-38 , am linken Auge 30-40 . (Siehe Fig. 5-8 S. 226 u. 227.) Es besteht nur noch Anästhesie der Stirn- und behaarten Kopfhaut. Alle übrigen Krankheitserscheinungen und Beschwerden - insbesondere traurige Verstimmung, Krämpfe, Anästhesie am übrigen Körper, Appetit- und Schlaflosigkeit - dauernd gewichen. Pat. befindet sich wohl. ${ }^{1}$ )

Wir haben es also hier mit einer hysterischen Erkrankung zu thun, wie selbe unzweifelhaft aus den Krampfzuständen, den hysteroepileptischen Anfällen, den Anästhesien in ibren wecliselnden Formen hervorgeht. Aber trotzdem bedarf es wohl - mit Rücksicht auf die Ausserordentlichkeit des, meines Wissens, in der Literatur einzigen Falles - einer besonderen Begriundung, dass diese Pupillen- und Accommodationslähmung hysterischer Natur war.

Da will ich nun hervorheben, dass diese Lähmung mit ihrer mässigen (nicht maximalen) Erweiterung der auf Licht, Accommodation und Convergenz nicht reagirenden Pupille das Bild der nuclearen Lähmung zeigte. Was sie aber von einer solchen Herderkrankung unter-

1) Seither habe ich mich zu wiederholten Malen überzeugt, dass dieser günstige Zustand bis gegenwärtig (2. März 1892) unverändert fortbesteht. 
22. November 1891. Pupillenreaction und Accommodation beiderseits normal.

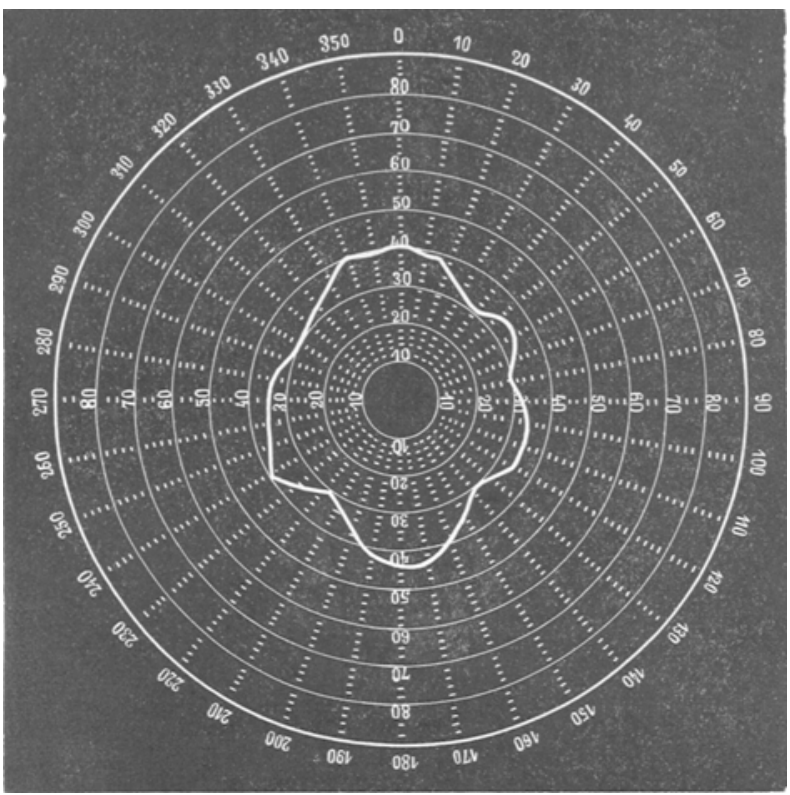

Fig. 5. Rechtes duge: Weiss.

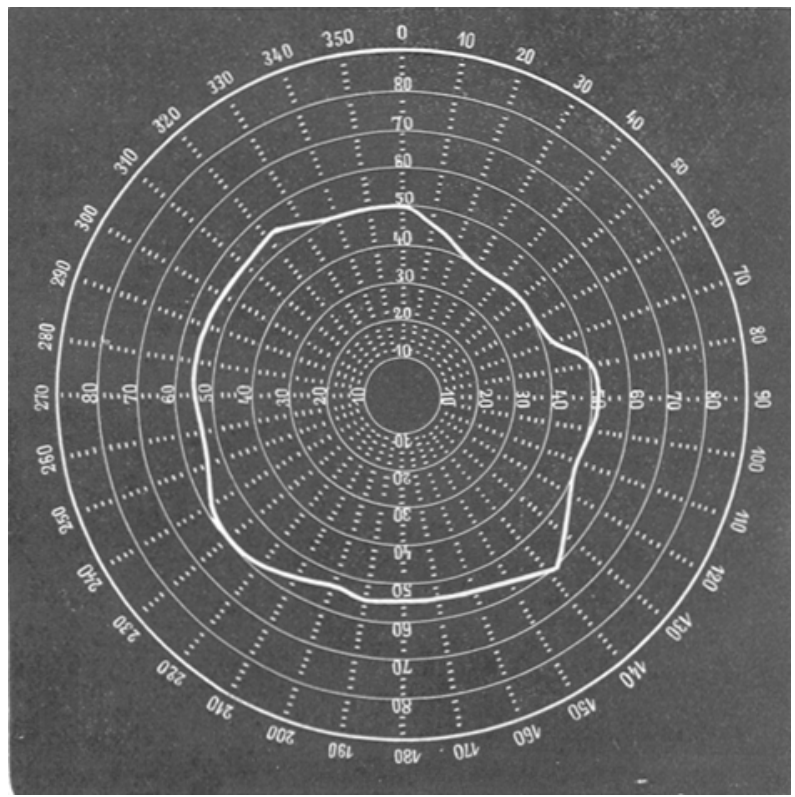

Fig. 6. Linkes Auge: Weiss. 
Hyster. Pupillen- u. Accommodationslähm., geheilt durch hypnot. Suggestion. 227

22. November 1891. Pupillenreaction und Accommodation beiderseits normal.

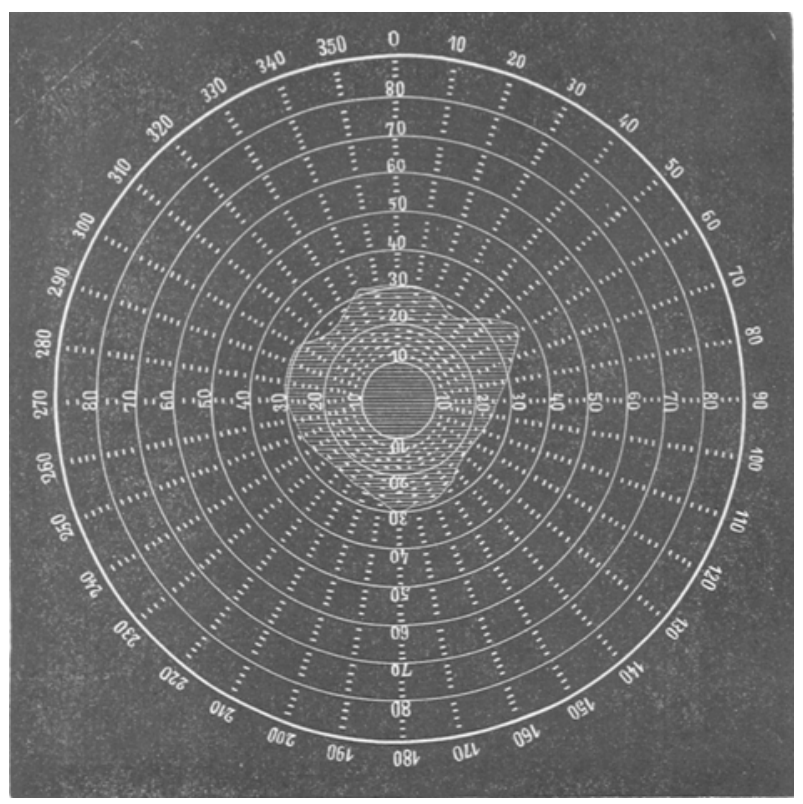

Fig. 7. Rechtes Auge: Roth.

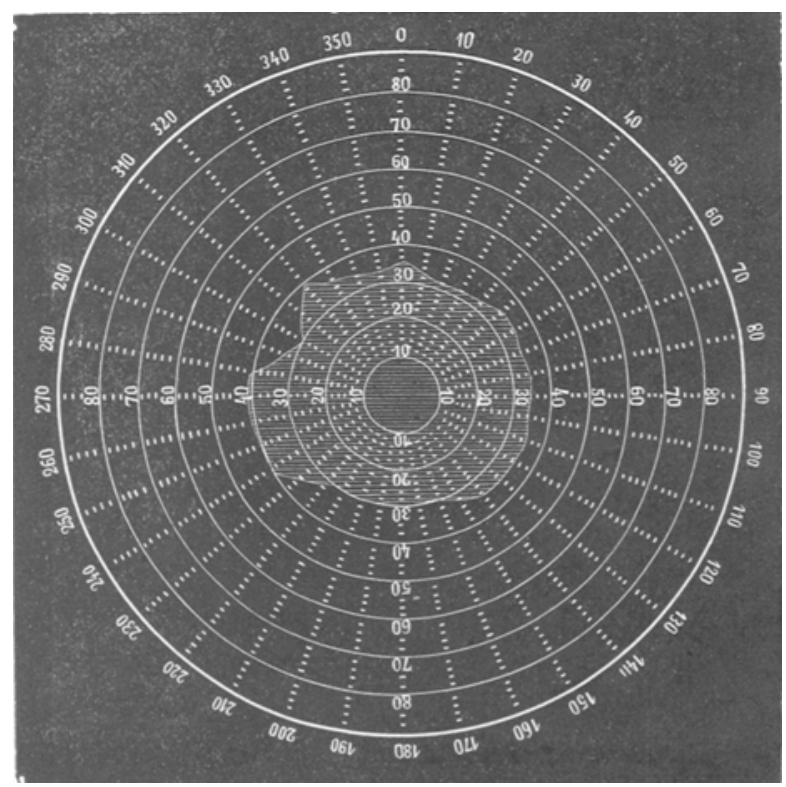

Fig. 8. Linkes Auge: Roth. 
schied, waren 1) die Anästbesien, und zwar in diesem Falle sowohl Hemianästhesien, welche mit der Lähmung der Pupille gleichzeitig auftraten und vergingen und mit ihr geradezu die Erscheinung des Tansfert zeigten, als auch dauernde Anästhesien (wie die der Stirn- und behaarten Kopfbaut), und 2) der rasche Wechsel in der Ausdehnungder Gesichtsfelder, welche sich genau nach dem Zustand der Pupillen richteten, mit deren Lähmung sofort hochgradig eingeschränkt wurden und mitderen Functionirung sich wieder erweiterten.

Der Sitz dieser Lähmungen des Sphincter iridis und M. ciliaris kann also nicht in den Oculomotoriuskernen am Boden des III. Hirnventrikels gesucht werden, sondern muss ebenso corticalen Ursprunges sein, wie die Anästhesien und Gesichtsfeldeinschränkungen, welche als Aeusserungen jener Functionsstörungen der Hirnrinde gelten, welche wir als Hysterie bezeichnen.

Aus dieser klinischen Beobachtung ergeben sich höchst interessante anatomisch-physiologische Folgerungen für den Sitz der Pupillenbewegung.

Bekanntlich reagirt die Pupille nicht nur auf Lichteinfall in das betreffende, sondern auch auf Lichteinfall in das andere Auge. Es muss also der Kern des Sphineter iridis in anatomischer Verbindung stehen mit den beiden NN. optici (mit dem der anderen Seite wahrscheinlich durch Verbindungsfasern mit dem Kern des Sphincter iridis der anderen Seite, mit welchem er synergisch wirkt). Desgleichen weist die synergische Reaction der Pupille mit der Accommodation und der Convergenzbewegung auf Verbindungen hin mit dem Kern des M. ciliaris und dem des M. rectus internus. Die Thatsache der hysterischen Pupillen- und Accommodationslähmung weist nun auch auf ein böheres corticales Centrum der Pupillenbewegung hin, ebenso wie wir es ftir die dem Willensact direct unterworfenen Accommodationsmuskeln und auch für die Gesichtsfeldperception annehmen müssen. $\mathrm{Zu}$ derselben Schlussfolgerung gelangte ich schon fruher auf anderem Wege gelegentlich meiner Beobachtungen über nucleare Pupillen- und Accommodationslähmungen. ${ }^{1}$ ) Daselbst bob ich die schon in frühen Stadien der Dementia paralytica auftretenden Unregelmässigkeiten und Ungleichheit der Pupillen hervor, sowie die Thierexperimente, insbesondere von D. Ferrier und FrançoisFranck, welche durch Reizung bestimmter Partien der Hirnrinde sowohl Erweiterung als Verengerung der contralateralen Pupille hervorrufen konnten.

1) Siehe meine Abhandlung: Ueber Ophthalmoplegia interna (Hutchinson). Wiener medicinische Presse 1891. Nr. 8 und 9. 
Demnach erscheint der Kern des Sphincter iridis als untergeordnetes Centrum, welches den Gipfelpunkt verschiedener Reflexbogen und den Knotenpunkt verschiedener associirter motorischer Nervenbahnen bildet, wäbrend das böhere Centrum der Pupillenbewegung. in der Hirnrinde zu suchen ist.

Was die Frage der Heilung dieser Pupillen- und Accommodationslähmung mittelst Hypnose anlangt, so nehme ich keinen Anstand, hier ein propter hoc und kein post hoc anzunehmen, weil auch die übrigen hysterischen Erscheinungen auf diese Weise gewichen sind. Wer sich mit hypnotischer Therapie befasst hat, weiss wohl, dass, wie wunderbare Heilungen bei functionellen Erkrankungen des Nervensystems auch erzielt werden, dieselben oft genug nicht mit einem Schlag $\mathrm{zu}$ bewerkstelligen sind, sondern wie andere therapeutische Agentien häufig längere Zeit fortgesetzt werden mïssen, bis man zum Ziele gelangt. Die therapeutische Suggestion des Arztes hat hier die ibr entgegenstehende Autosuggestion des Kranken zu überwinden, welch' letztere ja die Ursache der Krankheitserscheinungen ist. Die Dauer und Stärke verursacht es dann, dass es fortgesetzter suggestiver Einwirkung Seitens des Arztes bedarf, um die höheren Fähigkeiten: die Aufmerksamkeit, das Urtheil und den Willen wieder anzuregen und in die Bahnen zu den niedrigeren Centren der Vorstellungen und automatischen Bewegungen zu lenken, wo jene als Controlorgane ibren regulirenden Einfluss auszuiuben haben.

Zum Schluss will ich noch unter Anderem erwähnen, dass diese Kranke einen in der Hypnose von mir ertheilten sehr complicirten Auftrag am nächsten Tage zur angegebenen Stunde in autohypnotischem Zustande mit der grössten Pünktlichkeit und Sicherheit ausgeführt hat, wobei sie um die Mittagszeit die bevölkertsten Strassen unserer Stadt passiren und einen sehr umständlichen Einkauf für mich besorgen musste. Von allen diesen Dingen hatte sie nachträglich nicht die geringste Kenntniss. Auf Befehl verfasste und schrieb sie einen vollständig zusammenhängenden Aufsatz nieder, den sie in 2 hypnotischen Sitzungen, welche 3 und 6 Wochen später erfolgten, in correcter Weise fortsetzte. Durch Suggestion einer "Brandblase" an einer bezeichneten Stelle am Oberarm konnte ich eine Bulla von $11 \mathrm{Mm}$. Durchmesser hervorrnfen, welche etwa 3 Wochen zur Heilung brauchte, sowie ferner tropfenweises, 2 Stunden währendes Bluten aus der von mir bezeichneten Achselhöhle. - Dariiber und iiber eine Reihe anderer Beobachtungen und Untersuchungen gedenke ich, wie gesagt, später ausführlicher zu berichten. 